Documentation et bibliothèques

\title{
Défis et mutations : nouvelles compétences indispensables dans la formation archivistique belge
}

\section{Sébastien Soyez}

Volume 61, numéro 2-3, avril-septembre 2015

La formation en sciences de l'information

URI : https://id.erudit.org/iderudit/1032820ar

DOI : https://doi.org/10.7202/1032820ar

Aller au sommaire du numéro

Éditeur(s)

Association pour l'avancement des sciences et des techniques de la documentation (ASTED)

ISSN

0315-2340 (imprimé)

2291-8949 (numérique)

Découvrir la revue

Citer cet article

Soyez, S. (2015). Défis et mutations : nouvelles compétences indispensables dans la formation archivistique belge. Documentation et bibliothèques, 61(2-3), 125-128. https://doi.org/10.7202/1032820ar

Tous droits réservés (C) Association pour l'avancement des sciences et des techniques de la documentation (ASTED), 2015
Ce document est protégé par la loi sur le droit d'auteur. L'utilisation des services d'Érudit (y compris la reproduction) est assujettie à sa politique d'utilisation que vous pouvez consulter en ligne.

https://apropos.erudit.org/fr/usagers/politique-dutilisation/ 


\title{
Défis et mutations : \\ nouvelles compétences indispensables dans la formation archivistique belge
}

\author{
SÉBASTIEN SOYEZ \\ Archiviste-chef de travaux \\ Archives de l'État en Belgique, Service Préservation \& Accès numériques \\ Collaborateur scientifique \\ Université de Namur, Centre de recherche Information, Droit et Société (CRIDS) \\ sebastien.soyez@arch.be
}

\section{Genèse d'une mutation}

A u cours des dernières décennies, le développement de la société de l'information a généré une explosion informationnelle qui a métamorphosé les méthodes et les habitudes de travail dans la gestion et l'archivage de l'information. De nouveaux supports, et donc de nouvelles formes d'archives, sont apparus, appelant une gestion et une préservation adaptées à cette réalité. Les processus de travail se sont progressivement informatisés, avec des solutions en perpétuelle évolution au fil d'une technologie galopante. Ce phénomène s'amplifie et se complexifie de manière constante, conduisant nécessairement à des mutations de la profession archivistique, impliquant de nouvelles compétences, de nouvelles tâches et de nouveaux rôles.

Trop souvent, hélas, les archivistes sont absents de ce type de projet. Plusieurs raisons peuvent l'expliquer. La première est sans doute liée à une mauvaise compréhension du rôle de l'archiviste, qu'on associe erronément à la seule gestion des documents "inactifs ", c'est-à-dire au rangement d'un amas de documents papier poussiéreux dans une cave, une image désuète et peu flatteuse qui colle encore à la profession dans les milieux mal informés. La seconde raison vient peut-être des archivistes eux-mêmes qui, intimidés par le caractère complexe et technologique d'un projet innovateur, préfèrent ne pas s'en mêler, oubliant qu'ils pourraient contribuer utilement à la dimension de gestion de l'information.

Dans les cas où les archivistes sont impliqués dès les premiers travaux, il apparaît clairement que leur savoir-faire est un atout pour mener à bien un projet qui soit viable à long terme, envisageant tout le cycle de vie du document numérique et soucieux de préserver ses garanties d'authenticité, d'intégrité, de lisibilité et de pérennité. En outre, mieux que personne, l'archiviste connaît le contexte dans lequel les documents de son institution sont produits et reçus, les documents générés par les processus métier, de même que la circulation des documents entre les unités administratives. Il orchestre l'identification et le classement de l'information et consigne les étapes de création, de conservation et d'élimination de chaque document, dans des outils de gestion documentaire détaillés. L'archiviste possède des connaissances indispensables pour modéliser les processus et les flux informationnels sur la base desquels la solution sera développée, mais aussi pour contribuer à l'implantation de la solution, à la phase de test et à l'accompagnement des utilisateurs dans la gestion du changement.

Toutefois, pour pouvoir pleinement participer à la gestion et à la préservation de l'information numérique, il semble de plus en plus indispensable de doter l'archiviste de compétences additionnelles, qui sont encore souvent négligées dans les formations en archivistique. Les premières générations d'archivistes disposent bien sûr de connaissances spécifiques au regard du traitement d'archives traditionnelles ${ }^{1}$. Mais à l'ère du numérique, l'archiviste doit de plus en plus se préoccuper des documents dès leur création et, en conséquence, être capable de maîtriser l'information tout au long de son cycle de vie, quel que soit son support ou sa forme. Cette implication de l'archiviste dans la gestion dynamique et intégrée des documents ${ }^{2}$ appelle à un dialogue et à un rapprochement avec les autres disciplines, et en particulier les sciences et les technologies de l'information, la gestion et le droit.

Pour replacer la réflexion dans un contexte belge, la présente contribution se base sur une recherche préliminaire menée sur les principaux programmes de formation actuellement offerts en Europe francophone et

\footnotetext{
1. Par " archives traditionnelles ", on entend ici les archives créées sur un support traditionnel (principalement le papier) et qui peuvent être déchiffrées sans le secours d'un appareil. Ces archives comprennent également les archives iconographiques, les archives cartographiques et les archives photographiques (voir Roger Petit, Daniel Van Overstraeten, Herman Coppens \& Jacques Nazet. 1994. Terminologie archivistique en usage aux Archives de l'Etat en Belgique. Bruxelles : Archives générales du Royaume, p. 24).

2. La gestion intégrée des documents (ou GID) est la "gestion dans un processus unique de tous les documents [...], qu'ils soient technologiques ou analogiques, pendant tout leur cycle de vie, soit depuis leur création ou réception jusqu'à leur conservation permanente ou élimination». Elle met en œuvre un «système pérenne de gestion dont les composantes sont intégrées, afin de donner aux personnes accréditées accès à toute information pertinente portée par un document qui documente les activités d'une organisation " (voir la Politique de gestion intégrée des documents administratifs de Bibliothèque et Archives nationales du Québec, éditions 2009 et 2013, non publiées).
} 
au Québec ${ }^{3}$ et sur un article coécrit avec la professeure Marie Demoulin de l'EBSI .

\section{La formation classique en archivistique : constat et nécessaire évolution}

Depuis sa création, la formation classique en archivistique a visé à former des archivistes à la préservation de documents historiques sur supports analogiques. Comme l'archiviste est amené à traiter des documents produits dans un cadre temporel et géographique déterminé, il est utile qu'il puisse analyser leur contexte de création. Dès lors, ces formations proposent un découpage de l'apprentissage sur la base de périodes calquées sur celles de l'histoire : archivistes médiévistes et modernistes et archivistes contemporanéistes. Traditionnellement, cette formation classique les oriente vers des institutions de conservation du patrimoine documentaire publiques ou privées. On peut dès lors qualifier ces professionnels d'archivistes patrimoniaux.

Depuis la fin du $\mathrm{XX}^{\mathrm{e}}$ siècle, de nombreux programmes de formation archivistique classique ont connu une évolution sensible. Certes, ces changements ne sont pas perceptibles partout de la même manière. Dans de nombreux pays, surtout anglo-saxons, le virage a été amorcé il y a plus d'une décennie, notamment parce que les finalités de l'archivage patrimonial (archiving) et de l'archivage managérial ${ }^{5}$ (records management) sont déjà bien intégrées dans les pratiques professionnelles. Dans les pays francophones, et en particulier en Belgique, la prise de conscience a été plus lente, même si des évolutions positives sont perceptibles ces dernières années.

Le programme de formation archivistique classique est construit autour de socles de compétences ${ }^{6}$. L'acquisition de ces compétences peut varier d'un pays à l'autre, tantôt par le biais d'une filière spécifique au sein d'un master en histoire, tantôt par la voie d'un master dédié à l'archivistique ou encore par la voie d'un certificat spécialisé. Ces socles de compétences peuvent être regroupés en quatre catégories :

3. L'étude a porté sur les principaux programmes de niveau universitaire offerts en Belgique (francophone et néerlandophone), en France, en Suisse et au Québec, à partir des données disponibles sur les sites Web des institutions d'enseignement pour l'année académique 2014-2015. Les auteurs tiennent à remercier Ivan Barreau et Fiona Aranguren Celorrio pour leur précieuse collaboration dans la collecte de ces données.

4. Marie Demoulin \& Sébastien Soyez. 2014. Law and records management. In Archival Studies: New Skills for Digital Preservation. Proceedings of the $7^{\text {th }}$ Triennal Conference of DLM-Forum (12-14 November 2014), Lisbon.

5. Le terme " archivage managérial » est une des traductions du concept anglo-saxon de "records management " (</blog.cr2pa.fr/archivage-managerial/>). Notez que la traduction officielle du terme " records " retenue par ISO, notamment dans sa norme 15489 , est "documents d'activité ", ce qui pose dans la pratique des problèmes de compréhension. "Documents engageants ", « documents probants " sont à notre avis plus compréhensibles sur le terrain.

6. Les formations classiques les plus complètes proposent d'ailleurs des matières spécialisées pour chacune des périodes de l'histoire (Moyen-Âge, temps modernes et époque contemporaine).
- les matières rattachées à l'étude et à la gestion des archives en tant que telles, par exemple la théorie et la pratique archivistiques, ainsi que des cours plus spécifiques comme la diplomatique ou la paléographie;

- les matières dites historiques, qui permettent à l'archiviste de mieux appréhender le contexte de création des archives, comme l'histoire institutionnelle, sociale, économique, politique ou littéraire, l'histoire du droit, l'histoire de l'art et l'archéologie;

- les matières issues des sciences de l'information, qui permettent à l'archiviste de mieux maîtriser le support et le contenu informationnels des archives : la recherche documentaire, l'étude du livre et des bibliothèques, des médias;

- les matières périphériques issues d'autres sciences, comme la philologie, la littérature, le droit, pour ne citer que les principales.

Avec l'avènement des nouvelles technologies de l'information et de la communication, on constate que les formations archivistiques classiques commencent à s'adapter en conséquence, et à intégrer progressivement des cours plus techniques au sein des matières issues des sciences de l'information et d'autres sciences. Il s'agit par exemple de cours d'informatique, de management et de gestion de projet, d'analyse, de traitement et de gestion numérique des documents, des métadonnées, des formats et des supports de l'information numérique. Toutefois, à l'heure actuelle, cette tendance n'est pas encore généralisée.

Parallèlement à cette évolution, on constate qu'au sein de formations universitaires dédiées aux sciences et aux technologies de l'information et de la communication, des filières s'intéressent de plus en plus à la gestion documentaire et à l'archivistique. Mais ces évolutions s'effectuent de manière très différente d'un pays à l'autre, et parfois même d'une école, d'une université ou d'une communauté à d'autres dans un même pays, ce qui n'est pas sans créer quelques paradoxes sur le terrain professionnel. On peut en effet se demander s'il faut former distinctement les archivistes patrimoniaux et les records managers. Leurs fonctions sont malgré tout similaires, car

tous deux sont responsables de la préservation et de la communication des documents. Cependant, dans certains organismes et certains pays, il existe une distinction chronologique entre ces deux métiers, l'un s'attachant au document depuis sa création jusqu'à la fin de son usage administratif, l'autre le récupérant quand il est devenu archive historique. Tous deux présentent les mêmes compétences et les mêmes connais- 
sances, qu'il met [sic] en ouvre pour assurer la survie et l'intégrité des fonds. ${ }^{7}$

La réponse est-elle à trouver dans les besoins du terrain? Pour les archivistes patrimoniaux, les tâches restent sensiblement les mêmes, bien qu'ils soient de plus en plus souvent amenés à intervenir dans des projets de numérisation de fonds et à utiliser des outils informatiques durant toute la phase de traitement des archives. Mais pour les gestionnaires d'information qui œuvrent durant la phase dynamique des archives, les formations classiques proposées actuellement n'apportent pas toujours une réponse suffisante, même si une évolution est en cours. L'adaptation du cursus à l'environnement numérique et aux nouveaux besoins liés à l'archivage managérial (records management) s'opère de manière assez disparate, en particulier en Europe francophone. Une double évolution est donc nécessaire : sur le plan du contenu des formations, mais également sur le plan de l'organisation des filières à l'intérieur des programmes d'enseignement supérieur.

La situation belge s'inscrit dans cette conjoncture et n'est pas encore idéale. La formation archivistique reste à notre avis, et sur la base de notre analyse, étriquée dans des filières universitaires (master) dédiées quasi exclusivement à l'histoire ou, plus récemment, aux sciences de l'information. Certes, les premières ont encore en partie leur sens, car il est essentiel de transmettre des compétences en sciences historiques à de futurs archivistes qui traiteront des sources allant du Moyen-Âge à la période contemporaine. Mais d'autres compétences doivent être développées chez ces futurs spécialistes en gestion et préservation de l'information numérique. La partie néerlandophone du pays a déjà initié cette évolution : dans un premier temps, il y a plus d'une décennie, en associant quatre universités dans la conception d'un programme archivistique complémentaire à la formation classique en histoire; ensuite, en y intégrant progressivement des matières nouvelles, issues des sciences et des technologies de l'information. Cet exemple, ainsi que d'autres au Canada, en France ou en Suisse, montrent qu'il est possible de faire évoluer la formation classique afin d'ancrer la profession aux réalités du $\mathrm{XXI}^{\mathrm{e}}$ siècle et de répondre aux besoins prégnants du terrain.

\section{Nouveaux défis, nouvelles compétences}

Dès lors, une mutation progressive des programmes de formation en archivistique semble nécessaire pour permettre aux archivistes de participer pleinement à la gestion et à la préservation de l'information numérique, dans une perspective interdisciplinaire. Notre propos

7. Définition du métier d'archiviste reprise du site Web du Conseil international des archives (CIA), <www.ica.org/3835/que-sont-les-archives-et-le-mtierdarchiviste-/dcouvrir-les-archives-et-notre-profession.html>. n'est pas ici de dessiner le programme de formation archivistique idéal, ni d'entrer dans le débat sur la structure et l'organisation du cursus (certificat, baccalauréat, maîtrise, organisés en filières ou parcours, etc.) ou sur le contexte facultaire dans lequel de telles formations devraient être offertes (histoire, communication, bibliothéconomie, sciences de l'information, cours interfacultaires, interuniversitaires, etc.). Ces questions épineuses dépendent largement du contexte socioculturel, organisationnel, juridique et politique de l'enseignement supérieur et débordent le cadre du présent article.

Dans une optique plus globale, quel que soit le contexte, la présente contribution vise à souligner les compétences interdisciplinaires et les matières qui devraient être enseignées dès aujourd'hui pour que l'archiviste de demain maîtrise un corpus documentaire en profonde mutation. À cet égard, une attention particulière doit être portée à la gestion, au droit et aux technologies de l'information. En effet, quels que soient leur configuration et leur objet, les projets de gestion de l'information numérique et de gestion intégrée des documents mobilisent des compétences managériales, techniques et juridiques aux côtés de la gestion informationnelle et documentaire proprement dite et basée sur des principes archivistiques de tradition séculaire.

Notre réflexion s'appuie d'abord sur une expérience personnelle. Depuis 2013, nous nous impliquons activement dans un nouveau certificat interuniversitaire en management de l'information numérique en Belgique $^{8}$, basé sur un enseignement interdisciplinaire associant des cours de management, de gestion de projet et de gestion du changement, des cours de droit et des cours sur les technologies de l'information, directement en lien avec les besoins de la gestion de l'information numérique. Bien que la formation soit ouverte à une diversité de profils (juristes, informaticiens, managers, professionnels de l'information, etc.), la grande majorité des participants inscrits étaient issus du monde de l'archivistique ou de la documentation et ont exprimé un besoin de formation complémentaire en matière de gestion de l'information numérique pour pouvoir s'impliquer dans un projet au sein de leur organisme ou pour s'ouvrir à de nouveaux horizons.

Afin de relever ces nouveaux défis, qu'ils soient techniques, juridiques ou managériaux, l'archiviste de demain devra acquérir de nouvelles compétences qui viendront compléter son savoir-faire actuel. Il devra être capable de comprendre les enjeux et les choix technologiques de manière à pouvoir développer une stratégie de gestion et de préservation de l'information numérique en étroite collaboration avec les experts informatiques. En outre, il devra se positionner comme acteur à part entière dans un projet de gestion électronique de docu-

8. Ce certificat est organisé conjointement par l'Université de Namur, l'Université de Liège et les Archives de l'État en Belgique. Pour plus d'information, consulter $<$ www.docsafe.info $>$. 
ments pour accompagner le changement et orienter les pratiques dans une perspective d'archivage à court, moyen et long terme. Enfin, impliqué dès la phase dynamique des documents, il devra davantage prendre en compte les aspects juridiques liés notamment à la préservation de l'authenticité et de l'intégrité, à la transparence et à l'accessibilité ainsi qu'à la reproduction et à la diffusion de l'information. L'archiviste est ainsi appelé à jouer un rôle essentiel dans la chaîne de confiance entre le producteur d'un document et l'utilisateur, immédiat ou ultérieur.

Il va de soi que le but n'est pas de faire de l'archiviste un juriste, un informaticien ou un manager. L'archiviste doit demeurer un expert en sa matière et nous ne remettons pas ici en cause la nécessité absolue qu'il soit formé aux fondements de l'archivistique. L'objectif est davantage d'ancrer ces fondements au cœur d'une réalité multidisciplinaire. Cette interdisciplinarité accrue permet à l'archiviste de développer de nouvelles compétences, répondant aux besoins du terrain professionnel :

- compréhension des enjeux juridiques, techniques et organisationnels de la gestion de l'information, en particulier dans l'environnement numérique, durant tout son cycle de vie et quel que soit son support. Autrement dit, compréhension du contexte technique et socioculturel dans lequel s'inscrit la gestion de l'information dans la société numérique;

- compréhension du rôle clé qu'il doit jouer, en tant que professionnel de l'information, dans la gestion de l'information numérique. Sa formation doit lui donner les outils qui lui permettront de trouver sa place au sein d'un organisme et de s'affirmer dans un tel rôle;

- habileté à dialoguer avec les experts d'autres disciplines en vue de trouver des solutions communes et durables, qui prennent en considération l'ensemble des préoccupations liées à la gestion de l'information numérique;

- implication proactive dans les projets de gestion de l'information numérique au sein d'un organisme, dans le développement d'outils et de politiques de gestion de l'information numérique.

Il apparaît donc, de manière assez évidente, qu'une expertise spécifique et transversale est nécessaire pour l'archiviste de demain. Enrichir et diversifier ses compétences, c'est également permettre à l'archiviste de répondre à des besoins nouveaux tout au long du cycle de vie des archives. L'archiviste ne doit plus être cantonné à la fin de ce cycle, mais doit au contraire être actif durant toute la durée de vie d'une archive. Pour ce faire, il doit acquérir de nouveaux savoirs et développer de nouveaux savoir-faire, que l'on peut regrouper en trois catégories : des compétences en management et en gestion de projet, des compétences juridiques et des compétences techniques, en lien avec la gestion et la préservation de l'information numérique.

\section{Conclusion : un nouveau savoir-être de l'archiviste}

On pressent tout de suite l'importance cruciale de nouvelles compétences pour le développement d'un savoir-faire concret et utile dans la gestion et la préservation de l'information numérique. Mais de telles compétences s'avèrent également indispensables à l'acquisition d'un nouveau savoir-être, à l'heure où les archivistes se cherchent une nouvelle identité.

Moderniser les programmes de formation en archivistique, c'est montrer que la gestion de l'information est un métier d'avenir, pour susciter des vocations et des réorientations professionnelles, afin de suivre l'évolution des besoins et du marché de l'emploi. C'est également mettre en valeur, soutenir et encourager les professionnels à s'impliquer activement dans la gestion de l'information au sein de leur institution, avec une vraie valeur ajoutée.

Loin de sonner le déclin de la profession d'archiviste, la société de l'information se présente comme une opportunité à saisir. Le volume et la complexité de l'information sont tels que celui qui est capable de la gérer et de la préserver a une grande valeur dans la société du XXI ${ }^{\mathrm{e}}$ siècle, car, plus que jamais, l'information c'est le pouvoir. En tant que professionnel de l'information, l'archiviste a un rôle majeur à jouer sur ce terrain, moyennant l'acquisition de compétences additionnelles. Dès lors, il s'agit non seulement de se donner les moyens de former une nouvelle génération d'archivistes dans des programmes universitaires plus modernes et plus attrayants, mais également de compléter la formation des professionnels en quête de réorientation avec une offre de formation continue adaptée. (-) 